\title{
The fate of patients with acute aortic syndrome during the coronavirus (COVID-19) pandemic: A UK multicenter study
}

\author{
Ana Lopez-Marco, PhD, ${ }^{\mathrm{a}}$ Barbara Rosser, MD,${ }^{\mathrm{b}}$ Amer Harky, MD,${ }^{\mathrm{c}}$ Danilo Verdichizzo, MD, ${ }^{\mathrm{d}}$ \\ Iain McPherson, MD, ${ }^{\mathrm{e}}$ Emma Hope, RN, ${ }^{\mathrm{f}}$ Syed Qadri, MD,${ }^{\mathrm{g}}$ and Aung Oo, $\mathrm{MD},{ }^{\mathrm{a}}$ on behalf of the UK AS \\ Research Group
}

\section{ABSTRACT}

Objective: The coronavirus disease 2019 (COVID-19) pandemic has posed challenges to health care services across the world. There has been a significant restructuring of health care resources to protect services for patients with COVID-19-related illness and to maintain emergency and urgent medical and surgical activity. This study assessed access to emergency treatment, logistical challenges, and outcomes of patients with acute aortic syndrome during the early months of the COVID-19 pandemic in the United Kingdom.

Methods: This was a multicenter study, from March 1 to May 20, 2020 that included 19 cardiac centers, was a retrospective analysis of prospectively collected data obtained from individual centers' national cardiac surgical databases. Demographic details, choice of treatment, operative details, and outcomes were collected. COVID-19 screening, timing of surgery, and outcomes of COVID-19-positive and -negative patients were also analyzed.

Results: In total, 88 patients presented with acute aortic syndrome to participating centers from March 1 to May 20, 2020. There were 79 aortic dissections (89.8\%), 7 intramural hematomas $(7.9 \%)$, and 2 penetrating aortic ulcers $(2.3 \%)$. Seventy-nine patients $(89.8 \%)$ underwent surgery. In-hospital mortality was $25.3 \%(n=20)$. Postoperative complications included $13.9 \%$ postoperative stroke $(11.4 \%$ permanent and $2.3 \%$ temporary), $16.5 \%$ rate of hemofiltration, and $10.1 \%$ rate of tracheostomy. Nine patients were treated conservatively with a mortality of $60 \%$. Seven patients were diagnosed with COVID-19, and there was no associated mortality.

Conclusions: Despite extensive restructuring of health care resources, access to emergency and urgent treatment for patients with acute aortic syndrome was maintained in the early months of the COVID-19 pandemic in the United Kingdom. Clinical outcomes were similar to the prepandemic period. (JTCVS Open 2021;5:17-25)

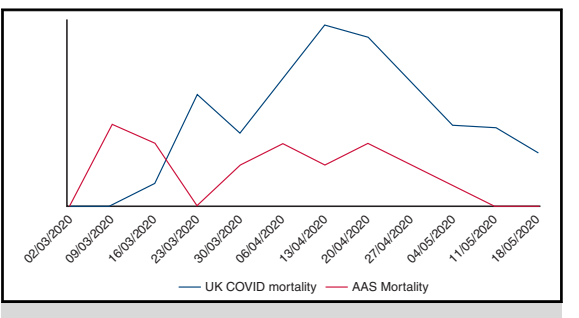

COVID-19 and surgical AAS mortality in the UK. COVID scale $=\times 100$ AAS mortality.

CENTRAL MESSAGE

The service provision for AAS was maintained during the early months of the COVID-19 pandemic in the United Kingdom with a very low rate of surgical turn down and COVID-19-related complications.

\section{PERSPECTIVE}

Although most patients were operated on an unknown COVID-19 status due to the natural history of AAS outweighing the risk of postoperative respiratory-related complications, only a minority of patients developed COVID-19 disease, which did not influence outcomes. With appropriate precautions, emergency surgery can be provided to patients with AAS with good outcomes during the COVID-19 pandemic.

See Commentaries on pages 26 and 28.

\footnotetext{
From the a Department of Cardiothoracic Surgery, St Bartholomew's Hospital, London; ${ }^{b}$ Department of Cardiothoracic Surgery, Royal Brompton and Harefield NHS Trust, London; ${ }^{\mathrm{c}}$ Department of Cardiothoracic Surgery, Liverpool Heart and Chest Hospital, Liverpool; ${ }^{\mathrm{d}}$ Department of Cardiothoracic Surgery, John Radcliffe Hospital, Oxford; ' Department of Cardiothoracic Surgery, Freeman Hospital, Newcastle; ${ }^{\mathrm{f}}$ Department of Cardiothoracic Surgery, Southampton University Hospital, Southampton; and ${ }^{\mathrm{g}}$ Department of Cardiothoracic Surgery, Blackpool Victoria Hospital, Blackpool, United Kingdom.

UK AS Research Group: Aung Oo, Geoffrey Tsang, Alex Cale, Jorge Mascaro, Mark Field, Manoj Kuduvalli, Giovanni Mariscalco, Jon Anderson, Sunil Bhudia, Ulrich Rosendahl, Jonathan Hyde, George Krasopoulos, Stefano Forlani, Karen Booth,
}

Uday Dandekar, Kelvin Lim, Reuben Jeganathan, Nidal Bittar, Mazyar Kanani, Hussein El-Shafei, and L. Balacumaraswani.

Received for publication Nov 8, 2020; accepted for publication Nov 17, 2020; available ahead of print Dec 17, 2020.

Address for reprints: Ana Lopez-Marco, PhD, St Bartholomew's Hospital, West Smithfield, London EC1A 7BE, United Kingdom (E-mail: ana.lopez-marco@ nhs.net).

2666-2736

Copyright $(C) 2020$ The Authors. Published by Elsevier Inc. on behalf of The American Association for Thoracic Surgery. This is an open access article under the CC BY-NCND license (http://creativecommons.org/licenses/by-nc-nd/4.0/).

https://doi.org/10.1016/j.xjon.2020.11.008 


\section{Abbreviations and Acronyms \\ AAS = acute aortic syndrome \\ COVID-19= coronavirus disease 2019 \\ CT $=$ computed tomography \\ NHS $\quad=$ National Health Service \\ PPE $\quad=$ personal protective equipment \\ SARS-CoV-2 $=$ severe acute respiratory syndrome coronavirus 2}

Video clip is available online.

The coronavirus disease 2019 (COVID-19) pandemic has affected all aspects of life of people across the globe to an unprecedented level. It has posed relentless challenges to the health care services of every country. Governments and health care systems were tasked with achieving a balance between public health care in containing severe acute respiratory syndrome coronavirus 2 (SARS-CoV-2) viral infection and maintaining acute medical services to reduce mortality secondary to acute cardiovascular emergency conditions. This has led to extensive reorganization of health care services in every region that involved protection of critical care facilities for treatment of COVID-19affected patients and deferring elective surgery. In parallel with this health care resource management, medical societies developed modified guidelines for patient selection and indications for emergency and urgent treatments. ${ }^{1-4}$

In the United Kingdom, like the rest of the world, all elective cardiac and aortovascular surgery was put on hold to protect patients' access to emergency and urgent surgery. Patients with acute aortic syndrome (AAS), having a devastating natural history with incremental hourly risk of mortality, require emergency surgery. ${ }^{5}$ In this study, we assessed the incidence, access to emergency treatment, logistical issues, as well as clinical outcomes of patients who presented with AAS during the early part of the COVID-19 pandemic in the United Kingdom.

\section{METHODS}

A multicentre service evaluation study was designed, and 19 centers across United Kingdom were recruited to assess the access for treatment, logistical limitations, and clinical outcomes of patients with thoracic aortic diseases during COVID-19 pandemic. The study period commenced from March 1, 2020, and patient recruitment is still ongoing at the time of data analysis for the initial experience (Video 1).

The 19 participating centers, grouped by regions, are London-St Bartholomew's Hospital, Royal Brompton and Harefield Hospitals and Hammersmith Hospital; Southeast-Royal Sussex County Hospital (Brighton), University Hospital Southampton and John Radcliffe Hospital (Oxford); West Midlands-Queen Elizabeth Hospital (Birmingham),

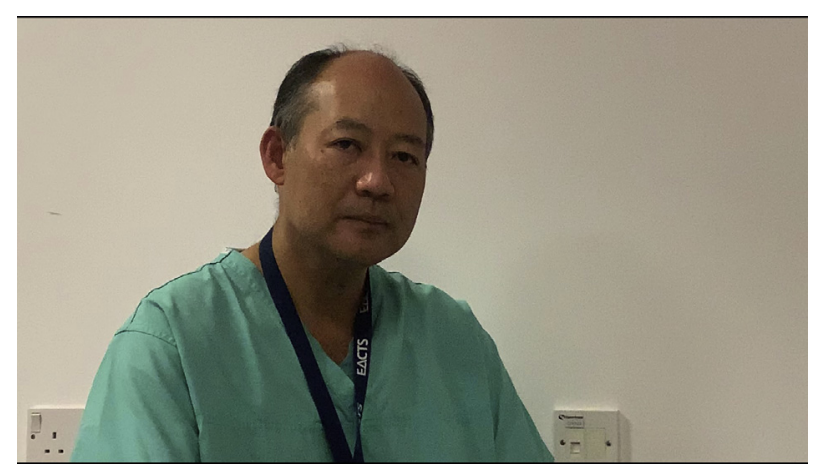

VIDEO 1. The senior author of the paper and principal investigator of the study, Professor A. Oo, explains the relevance of the study and summarizes its main results. Video available at: https://www.jtcvs.org/article/S26662736(20)30149-2/fulltext.

University Hospital Coventry and Royal Stoke University Hospital; East Midlands-Glenfield Hospital (Leicester); Northwest-Liverpool Heart and Chest Hospital and Blackpool Victoria Hospital; Yorkshire and Humberside-Sheffield Teaching Hospital and Castle Hill Hospital (Hull); Northeast-Freeman Hospital (Newcastle) and James Cook University Hospital (Middlesbrough), Scotland-Royal Infirmary of Edinburgh and Aberdeen Royal Infirmary, and Northern Ireland-Royal Victoria Hospital (Belfast). These centers represent $66 \%$ of the aortic units in the country, including the largest specialized aortic centers and cover most of the geographic areas. The other 15 aortic units declined to participate in the study for different reasons, including inability to provide emergency surgery cover during the COVID-19 pandemic, insufficient resources to collect the data, and/or individual preferences.

In this substudy, we evaluated access to emergency and urgent treatment, logistical limitations, and clinical outcomes of patients presented with AAS to participating centers from March 1 to May 20, 2020.

This was a retrospective analysis of prospectively collected data obtained from the National Cardiac databases of the individual centers. Details about preoperative demographics and risk factors, type of AAS, DeBakey classification, treatment of choice, operative details, outcomes, and postoperative complications were obtained from National Cardiac Databases of the individual centers. A detailed analysis of the COVID19 screening perioperatively and its effect on decision-making and timing of the treatment were also analyzed. The anonymized patient data from individual centers were transferred securely to St Bartholomew's Hospital for data cleaning and analysis. Data analysis was performed with SPSS, version 25 (IBM Corp, Armonk, NY).

Ethical approval was obtained from each participating center after acceptance of the study protocol at the recruiting center (St Bartholomew's Hospital). Individual patient consent was waived by the local Ethical Research Board due the anonymized nature of the data.

\section{Modification of Guidelines for Patient Selection and Management for Patients With Aortovascular Conditions During the COVID-19 Pandemic}

A modified protocol for patient selection and treatment of aortovascular conditions during the COVID-19 pandemic was developed on March 25, 2020, following a multidisciplinary team consultation involving cardiac surgeons, vascular and endovascular surgeons, interventional radiologists, cardiologists with interest in aortopathy, clinical geneticists, anesthetists, and intensivists at St Bartholomew's Hospital. It was developed after reviewing all existing clinical guidelines from international clinical societies and also taken into consideration of the potential increased risk of treatment if patient were to be infected with SARS-CoV-2 perioperatively. ${ }^{6}$ It was 
endorsed by the UK Aortic Surgery Group and the Society for Cardiothoracic Surgery.

The protocol defines the cohort of aortovascular patients eligible for referral and treatment during the COVID-19 pandemic, triaged in several categories depending on the level of urgency at time of referral/presentation. Patients with AAS and ruptured aneurysms of any anatomical location were categorized as emergency conditions, and it was agreed that they should be accepted and operated on at the earliest opportunity, including out-of-hours, due to the increased risk of mortality while waiting. ${ }^{5}$ This modified protocol was widely adopted in all participating centers.

\section{Preoperative COVID-19 Screening}

On March 26, 2020, a preoperative screening protocol for SARS-CoV-2 virus was introduced at St Bartholomew's Hospital after multidisciplinary review of available evidence and Public Health England guidance. ${ }^{7}$ It included a combination of 2 negative nasopharyngeal swabs for polymerase chain reaction for ribonucleic acid analysis, a noncontrast computed tomography (CT) of the thorax to assess changes in the lung parenchyma suggestive of COVID-19 disease, and analysis of the lactate dehydrogenase levels and lymphocyte counts. This screening protocol was disseminated through the Society for Cardiothoracic Surgery to the rest of United Kingdom and was adopted widely. ${ }^{8,9}$

However, in the case of patients with AAS requiring emergency surgery, although nasopharyngeal swabs were taken on admission to cardiac surgical centers, the surgical teams proceeded without waiting for results. This was due to the balanced decision that the incremental time delay-related increased risk of mortality was more significant than the risk of increased perioperative complications from COVID-19 infection. ${ }^{8,9}$

Irrespective of the patient's COVID-19 status, the surgical procedures and postoperative care in intensive therapy unit environments were performed using universal measures of personal protective equipment $(\mathrm{PPE})^{10}$ and with a limited number of theater staff in contact with the patient before endotracheal intubation (consultant anesthetist, anesthetic trainee, and operative department practitioner wearing full PPE) as well as a 20-minute period before other theater staff could enter the theater and start to allow the air in theater to be recycled to reduce the viral load. This practice precaution was to protect staff members from undiagnosed infection as well as to protect patients from staff, given the fact that staff were not routinely tested for this virus.

\section{RESULTS}

In total, 189 patients with aortovascular conditions were admitted to the 19 participating centers between March 1 and May 20, 2020. There were 88 patients who presented with an AAS and were the focus of this substudy.

AAS was categorized per subtypes: aortic dissection $(\mathrm{n}=79,89.8 \%)$, intramural hematoma $(\mathrm{n}=7,7.9 \%)$, and penetrating aortic ulcer $(\mathrm{n}=2,2.3 \%)$ and by anatomical locations: DeBakey I $(\mathrm{n}=71,81 \%)$, DeBakey II $(\mathrm{n}=7,8 \%)$, and DeBakey III $(\mathrm{n}=10,11.4 \%)$. (Figure 1$)$.

\section{Surgically Treated Group}

In total, 79 patients $(89.8 \%)$ were treated with surgery. Mean age of this cohort was 62 years (range, 29-83 years) and $32.9 \%(\mathrm{n}=26)$ were female. Rest of comorbidites and cardiovascular risk factors are listed in Table 1. The surgical procedures performed were aortic valve and ascending aorta replacement $(n=6)$, aortic root replacement $(n=32)$, ascending aorta and hemiarch replacement $(\mathrm{n}=23)$, total arch replacement $(n=18)$, frozen elephant trunk repair of descending thoracic aorta $(\mathrm{n}=7)$, descending thoracic aorta replacement $(\mathrm{n}=4)$, and thoracic endovascular aortic repair $(\mathrm{n}=2)$.

Surgery was offered predominantly as emergency during the same day of the admission $(\mathrm{n}=55,69 \%)$ or the following day $(\mathrm{n}=12,15.2 \%)$. In another 12 cases, the operation was performed within 15 days of the admission (mean 7.4 days, range 2-15 days) due to progression of the presenting disease (ie, expanding intramural hematomas or penetrating ulcers, complicated DeBakey III aortic dissection), the need for surgical planning (ie, debranching + thoracic endovascular aortic repair) or

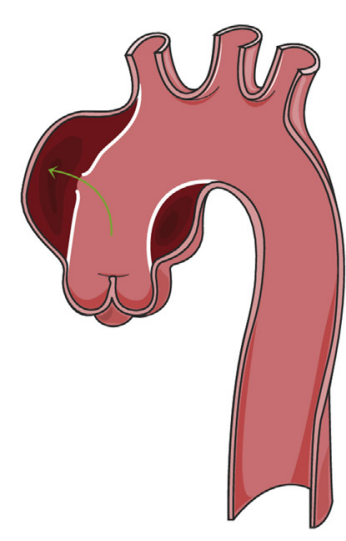

De Bakey I
$\mathrm{n}=71$

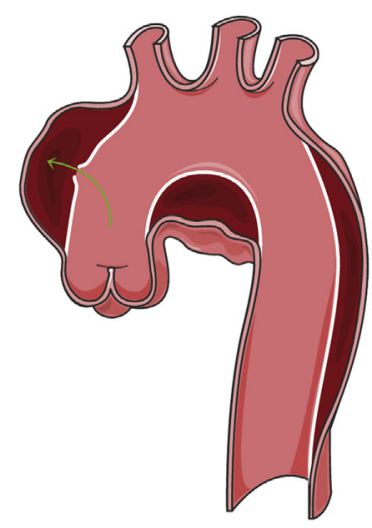

De Bakey II

$\mathrm{n}=7$

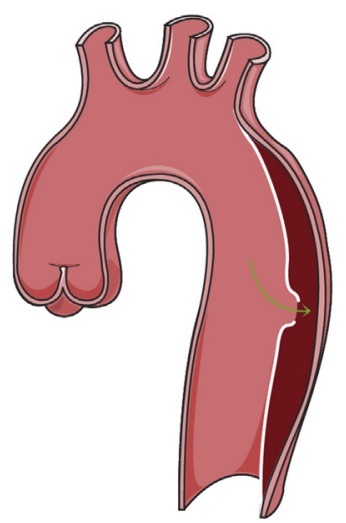

De Bakey III
$\mathrm{n}=10$

FIGURE 1. Number of patients that presented with an acute aortic syndrome to the participating centers and were included in the study, according to the DeBakey anatomical classification: DeBakey I (affecting the ascending aorta, arch and descending aorta, $\mathrm{n}=71$ ), DeBakey II (affecting only the ascending aorta, $\mathrm{n}=7$ ) and DeBekay III (confined to the descending aorta, $\mathrm{n}=10$ ). Reprinted with permission from Servier Medical Art. 
TABLE 1. Demographics and preoperative risk factors

\begin{tabular}{|c|c|c|}
\hline & n/Mean (range) & $\%$ \\
\hline Age & $62(29-83)$ & - \\
\hline Female sex & 26 & 32.9 \\
\hline Hypertension & 59 & 74.7 \\
\hline Diabetes & 6 & 7.6 \\
\hline Hypercholesterolemia & 12 & 15.2 \\
\hline COPD & 7 & 8.9 \\
\hline Creatinine & $97.7(42-288)$ & - \\
\hline Dialysis & 1 & 1.3 \\
\hline Ex-smoker & 12 & 15.2 \\
\hline Current smoker & 13 & 16.5 \\
\hline Previous stroke & 2 & 2.5 \\
\hline Previous TIA & 4 & 5.1 \\
\hline Peripheral vascular disease & 7 & 8.9 \\
\hline Previous myocardial infarction & 5 & 6.3 \\
\hline Previous PCI & 1 & 1.3 \\
\hline Atrial fibrillation & 8 & 10.1 \\
\hline Poor EF & 3 & 3.8 \\
\hline Moderate EF & 6 & 7.6 \\
\hline Previous cardiac surgery & 7 & 8.9 \\
\hline Previous aortic surgery & 5 & 6.3 \\
\hline Previous endovascular treatment & 2 & 2.5 \\
\hline EuroSCORE II & $9.6(1.8-40.8)$ & - \\
\hline
\end{tabular}

COPD, Chronic obstructive pulmonary disease; TIA, transient ischemic attack; $P C I$, percutaneous coronary intervention; $E F$, ejection fraction; EuroSCORE, European System for Cardiac Operative Risk Evaluation.

waiting for the COVID-19 screening results in urgent patients (Table 2).

In-hospital mortality was $25.3 \%(\mathrm{n}=20)$, with a $10.1 \%$ rate of intraoperative deaths $(n=8)$. Cause of death were categorized as follows: cardiac $(\mathrm{n}=9,45 \%)$, multiorgan failure $(\mathrm{n}=5,25 \%)$, stroke $(\mathrm{n}=3,15 \%)$, abdominal

TABLE 2. Postoperative complications

\begin{tabular}{lc}
\hline & $\mathbf{n}(\%)$ \\
\hline Intraoperative death & $8(10.1)$ \\
Hospital death & $20(25.3)$ \\
Reintubation & $5(6.3)$ \\
Tracheostomy & $8(10.1)$ \\
\hline Bleeding/tamponade & $9(11.4)$ \\
GI bleeding & $0(0.0)$ \\
Mesenteric ischemia & $2(2.5)$ \\
Stroke & $11(13.9)$ \\
Myocardial infarction & $0(0.0)$ \\
Renal failure & $18(22.8)$ \\
\hline Hemofiltration & $13(16.5)$ \\
Atrial fibrillation & $26(32.9)$ \\
\hline GI, Gastrointestinal. &
\end{tabular}

ischemia $(\mathrm{n}=2,10 \%)$, and aortic rupture $(\mathrm{n}=1,5 \%)$. Postoperative complications included $13.9 \%$ postoperative stroke (11.4\% permanent and $2.3 \%$ temporary), $16.5 \%$ rate of hemofiltration, and $10.1 \%$ rate of tracheostomy. Mean length of mechanical ventilation time was 71.5 hours (30 minutes to 53 days), and mean length of intensive therapy unit stay was 4.8 days ( 2 hours to 53 days).

\section{Nonsurgical Treatment Group}

A total of 9 patients were not managed surgically due to a variety of reasons. Three patients were accepted for emergency surgery but died either waiting for transfer or en route to the specialized center or during the anesthetic induction due to aortic rupture and cardiac tamponade. Mean age was 63.3 years (range, $45-81$ years).

Five patients were denied emergency surgery due to clinical complexity. Three of them had a history of previous cardiac or aortic surgery, and 1 of them had signs of COVID-19 on the preoperative CT scan. Mean European System for Cardiac Operative Risk Evaluation II was $16.9 \%$. Two patients were discharged alive; hence, the mortality for this group was $60 \%$.

Another patient was admitted with a noncomplicated DeBakey III aortic dissection and did not require surgical treatment. He was diagnosed with COVID-19 during the hospital admission but did not develop any symptoms or respiratory complications and was discharged home.

\section{Regional and Temporal Variation in Presentation of AAS}

There was a clear regional variation in the presentation of the AAS (Table 3), with the London region accumulating the greatest number of cases, even exceeding the prepandemic activity. This variation is due to the creation of the Pan London Emergency Cardiac Surgery Service, which concentrated all the cardiac and aortic surgical activity for the region in 2 of the participating centers (St Bartholomew's Hospital and Brompton and Harefield National Health Service [NHS] Trust), designed as COVID-19-free environments $^{11}$ (Figure 2). The majority of regions have managed to maintain the same activity compared with the prepandemic period (Figure 3). There was a clear impact of the start of the lockdown in the UK, with no patients with AAS being admitted to the hospital during the first week of the lockdown followed by the activity exponentially increasing immediately after and reaching a plateau by the end of April 2020 (Figures 4 and 5, Video 1).

\section{Patients With COVID-19}

A total of 7 patients were diagnosed with COVID-19 in the perioperative period. Five were diagnosed in the postoperative period, having been operated on with unknown COVID-19 status due to the clinical emergency. The diagnosis of COVID-19 with positive swab was made between 
TABLE 3. Number of patients with acute aortic syndromes operated in each of the participating centers during the study period (pandemic activity) and during the equivalent months before the pandemic (March to May 2019; prepandemic activity)

\begin{tabular}{lcc}
\hline \multicolumn{1}{c}{ Centre } & $\begin{array}{c}\text { Pandemic } \\
\text { activity }\end{array}$ & $\begin{array}{c}\text { Prepandemic } \\
\text { activity }\end{array}$ \\
\hline St Bartholomew's Hospital & $27(34.2 \%)$ & $14(17.5 \%)$ \\
\hline Royal Brompton and Harefield NHS Trust & $8(10.1 \%)$ & $6(7.5 \%)$ \\
\hline Hammersmith Hospital & 0 & $3(3.7 \%)$ \\
\hline Royal Sussex County Hospital & $1(1.3 \%)$ & $5(6.2 \%)$ \\
\hline University Hospital Southampton & $6(7.6 \%)$ & $5(6.2 \%)$ \\
\hline John Radcliffe Hospital & $6(7.6 \%)$ & $6(7.5 \%)$ \\
\hline Queen Elizabeth Hospital & 0 & $3(3.7 \%)$ \\
\hline University Hospital Coventry & $1(1.3 \%)$ & $2(2.5 \%)$ \\
\hline Royal Stoke University Hospital & $1(1.3 \%)$ & 0 \\
\hline Glenfield Hospital & $2(2.6 \%)$ & $1(1.2 \%)$ \\
\hline Liverpool Heart and Chest Hospital & $7(8.9 \%)$ & $9(11.2 \%)$ \\
\hline Blackpool Victoria Hospital & $4(5.1 \%)$ & $2(2.5 \%)$ \\
\hline Sheffield Teaching Hospital & $6(7.6 \%)$ & $3(3.7 \%)$ \\
\hline Castle Hill Hospital & $2(2.6 \%)$ & $2(2.5 \%)$ \\
\hline Freeman Hospital & $5(6.3 \%)$ & $4(5 \%)$ \\
\hline James Cook University Hospital & $1(1.3 \%)$ & $2(2.5 \%)$ \\
\hline Royal Infirmary of Edinburgh & $3(3.4 \%)$ & $4(5 \%)$ \\
\hline Aberdeen Royal Infirmary & 0 & $1(1.2 \%)$ \\
\hline Royal Victoria Hospital Belfast & $3(3.4 \%)$ & $4(5 \%)$ \\
\hline$N H S$, National Health Service. & & \\
\hline
\end{tabular}

postoperative days 2 and 24 (mean day 10). Only 1 of these patients required prolonged mechanical ventilation, but there was no COVID-19-related mortality.

One patient had signs of COVID-19 disease in the preoperative CT despite being asymptomatic and swab negative. He required reintubation and ventilation 7 days after surgery and died of respiratory failure. The last patient who tested positive for COVID-19 on admission was treated conservatively due a noncomplicated DeBakey III aortic dissection and did not develop any COVID-19-related complications.

\section{DISCUSSION}

The COVID-19 pandemic has affected health care services to an unprecedented scale globally. In the United Kingdom, the NHS was reorganized across all regions with the guidance of Public Health England and the Central Government, reducing or even stopping the elective surgical activity in most of the regions after the declaration of the lockdown on March 23, 2020. , $10^{2}$

There was a clear impact at the start of the lockdown, with no patients with AAS being admitted to hospital during the first week of the lockdown. This reflects the fear and anxiety of the general population not presenting their illness to Accident and Emergency services. Although there was an exponential increase in AAS admissions during the following weeks in April, it took 3 weeks before activity reaching to a prelockdown level in the United Kingdom.

In London, in comparison with the prepandemic period, overall the service provision for AAS has been maintained by concentrating all the aortic surgical activity for the region as part of the Pan London Emergency Cardiac Surgery Service. ${ }^{11}$ However, the activity in the majority of individual centers were just below the number of AAS cases operated during the same equivalent period last year. This was also supported by the adoption of the modified clinical guidelines for patient selection and indications for surgery. ${ }^{1-4,6}$ This has protected access of patients with aortovascular conditions for emergency and urgent surgery. It has certainly posed a challenge to achieve a balance between protection the patients with AAS versus protection of NHS for the rest of the population.

The result of the COVID-19 screening was not awaited in the majority of the patients with AAS, as it was considered that the risk of mortality while waiting for the swab results exceeded the risk of developing COVID-19-related complications in the immediate postoperative period. ${ }^{5}$ However, the number of patients in this cohort diagnosed with COVID-19 in the immediate postoperative period was minimal $(n=7)$, with the majority of those who were diagnosed in the early weeks of the study and at least 1 week after the operation, whilst already in contact with the general population. There was no COVID-19-related mortality in this group. The most likely explanation for this is after the declaration of the lockdown, the patients presenting in hospital with AAS were already isolating at home and were shielded in hospital only in contact with staff wearing full PPE measures, and those factors contributed to the low rate of postoperative COVID-19 infection compared with other surgical series. ${ }^{12}$

The proportion of patients with AAS treated surgically during this period of the pandemic reached almost $90 \%$, against initial predictions where planned surgical activity could have been severely affected by lack of intensive care beds, shortage of intensive care nurses, the fear of the health care professionals to expose themselves to aerosol-generated procedures, or the uncertainty of level of protection ensured by PPE measures.

During this early part of COVID-19 pandemic, there was a shortage of PPE in NHS hospitals. There has been uncertainty in the level of protection provided by PPE for both patients as well as health care workers. Moreover, operating such long surgical procedures wearing full PPE in theaters with restricted staff flow has a significant impact on the comfort zone of the team as a whole. The effect of FFP3 (ie, filtering face piece class 3 ) masks (and with surgical 
Acute aortic syndromes
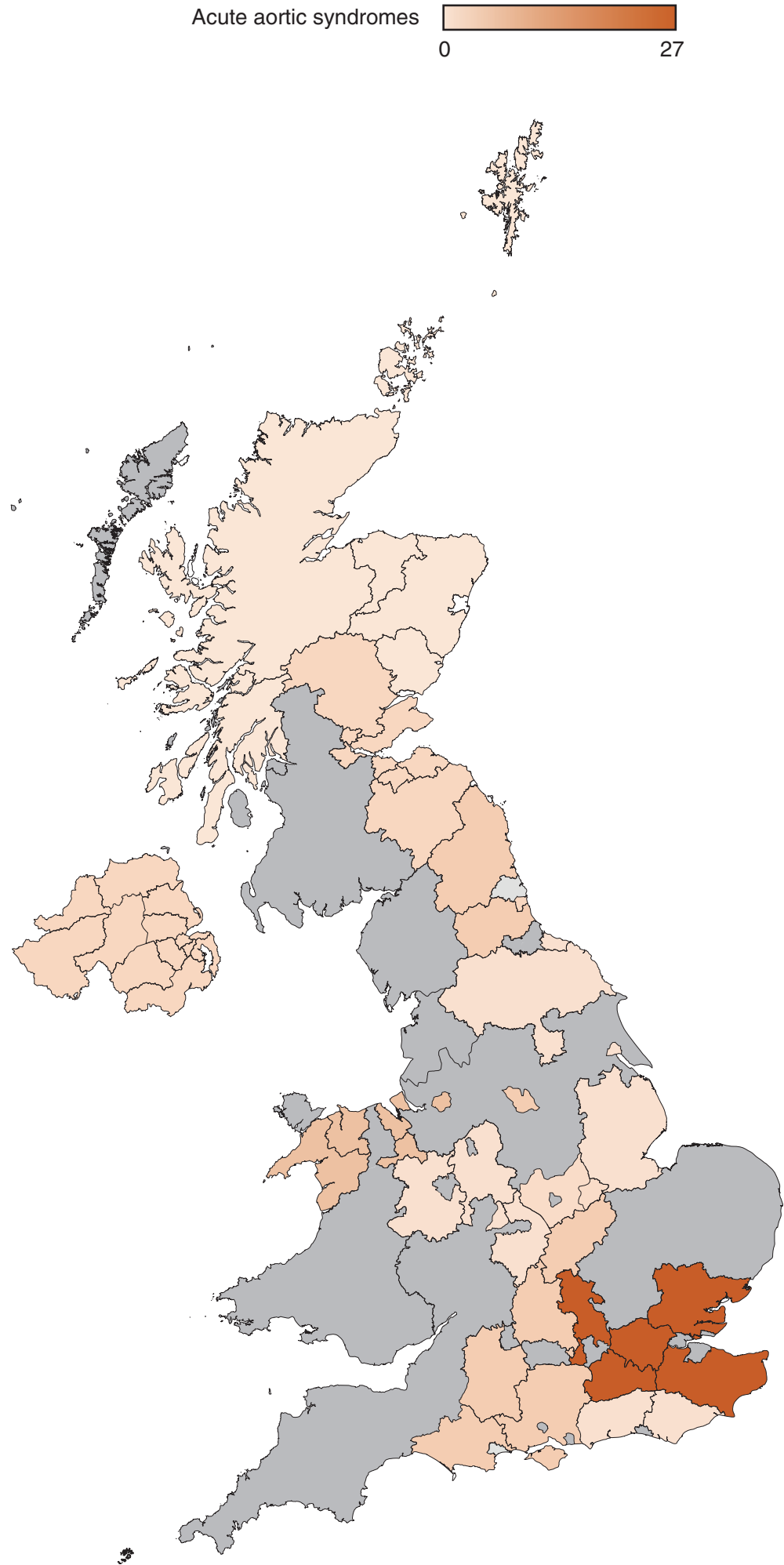

FIGURE 2. Geographic variation in presentation of acute aortic syndromes to hospital during the study period in the 19 participating centers in the United Kingdom. The graded colors represent the number of patients with acute aortic syndromes that were admitted to hospital for assessment and/or surgical treatment according each geographical region. The areas displayed in gray were the regions covered by centers not contributing to the study. 
2019

Acute aortic syndromes
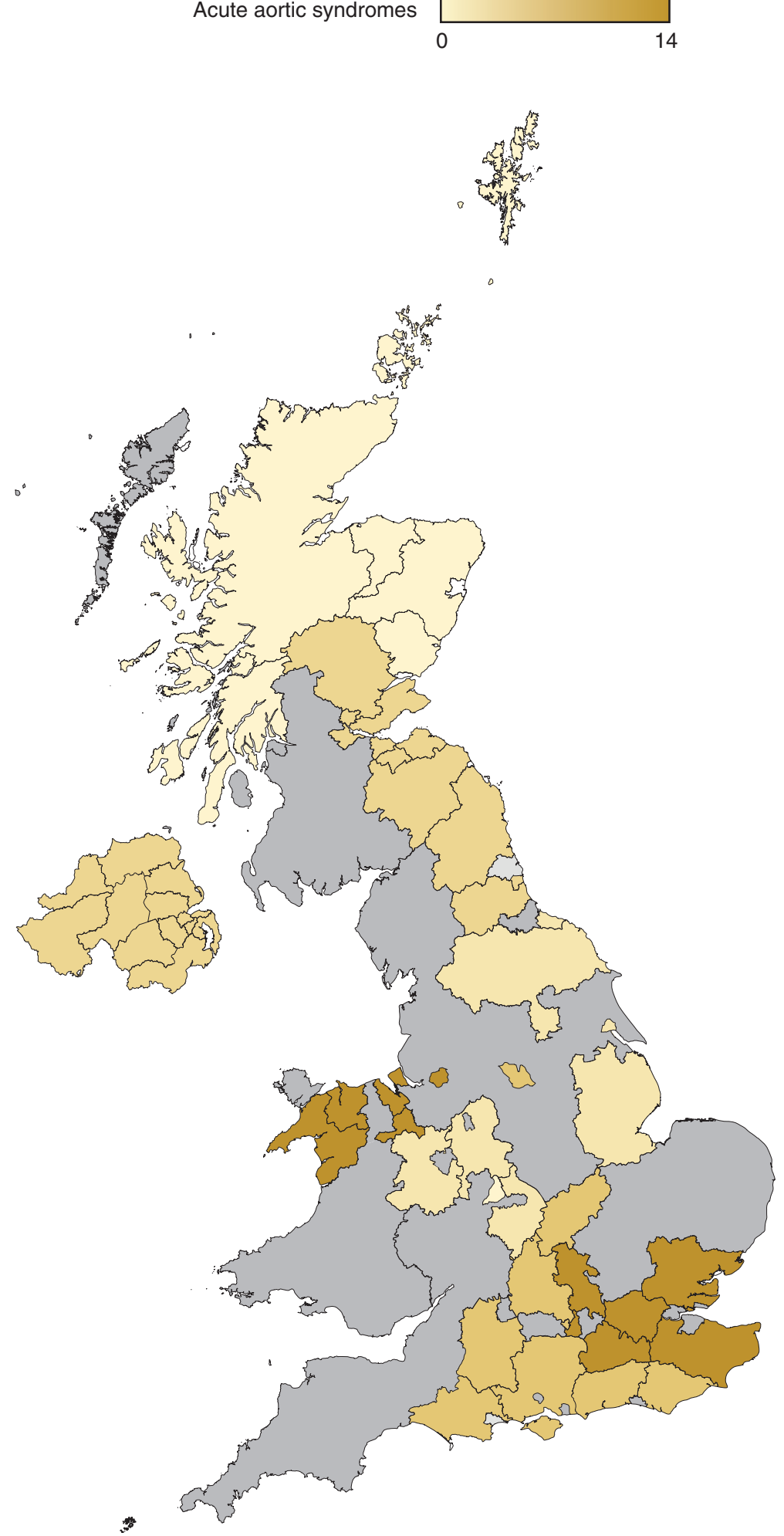

FIGURE 3. Geographic variation in presentation of acute aortic syndromes to hospital in the 19 participating centers in the United Kingdom during the equivalent months to the study period but the previous year (2019). The graded colors represent the number of patients with acute aortic syndromes that were admitted to hospital for assessment and/or surgical treatment according each geographical region, showing similar activity when compared with the pandemic period displayed in Figure 2. The areas displayed in gray were the regions covered by centers not contributing to the study. 


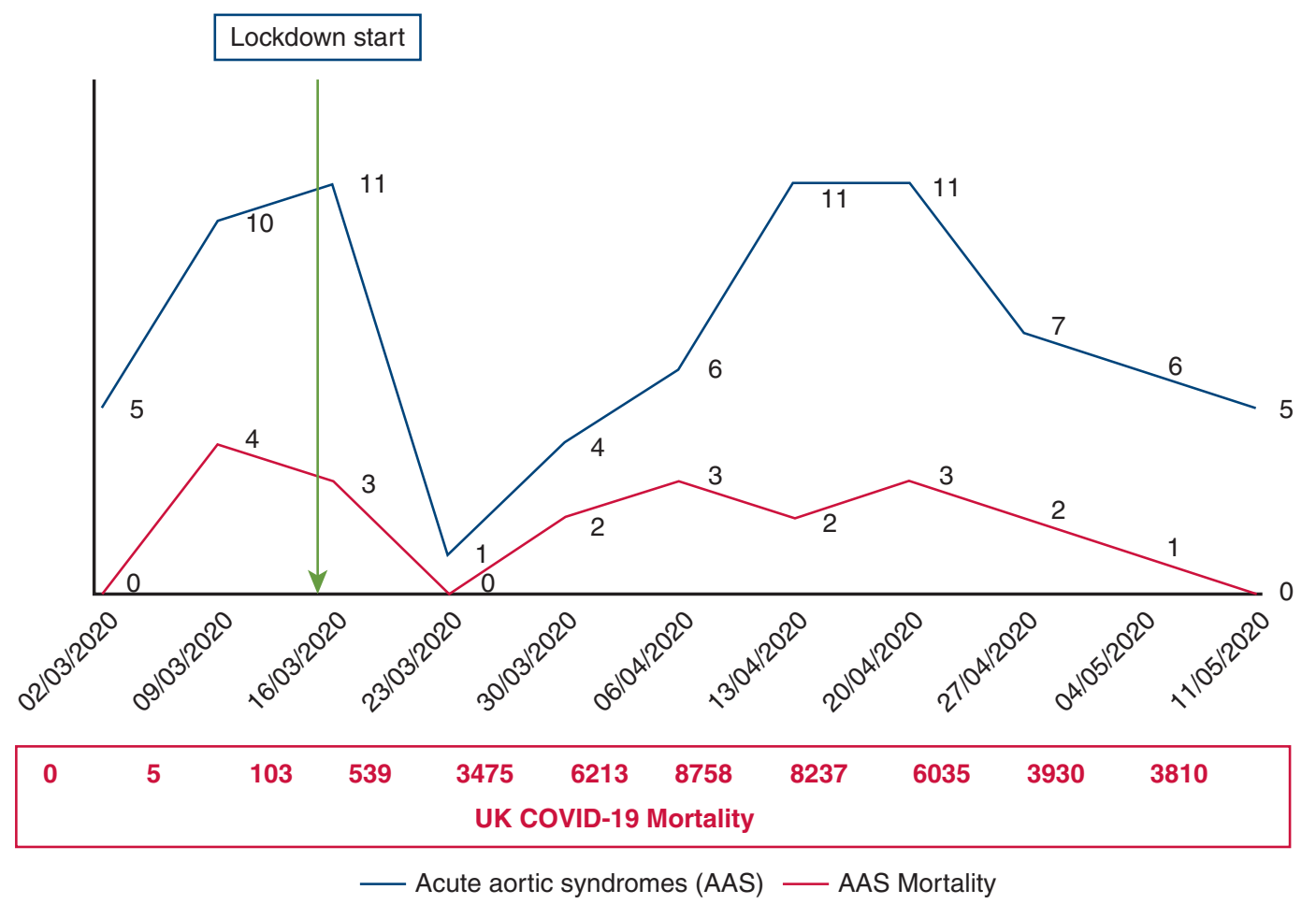

FIGURE 4. Time variation in presentation of acute aortic syndromes to hospital during the study period in the 19 participating centers in the United Kingdom. The vertical green arrow marks the start of the lockdown in the United Kingdom. The blue line displays the weekly number of patients with acute aortic syndromes admitted to the participating centers, noticing a clear reduction after the start of the lockdown with a progressive recovery of the activity in the following weeks. The red line displays the mortality of patients with acute aortic syndromes admitted to hospital. Below the $\mathrm{x}$-axis, there is a numerical display of the overall number of deaths due to COVID-19 in the United Kingdom weekly. Note the exponential increase in COVID-19 deaths coincides with a reduction of presentation in aortic syndromes. COVID-19, Coronavirus disease 2019.

mask as an additional outside layer) and visors on the

Surgical mortality of this cohort was $25 \%$, which is routine team communication as well as physical exhaustion similar to the reported outcomes before the pandemic., ${ }^{5,13,14}$ of staff cannot be underestimated.

This is worthy of note, as the multidisciplinary teams were

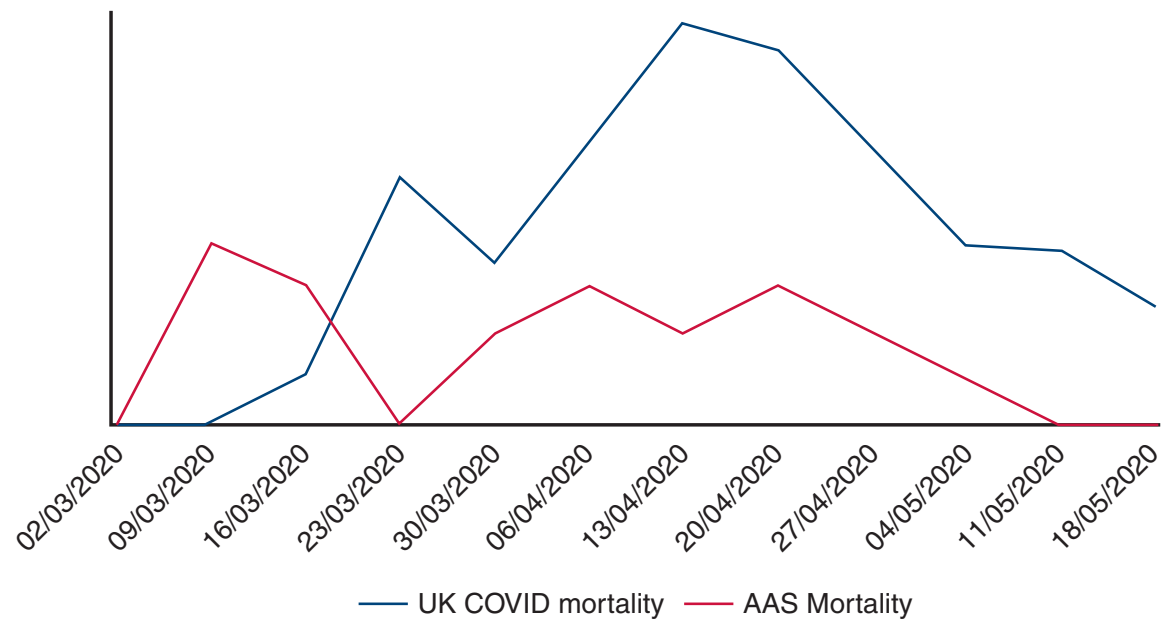

FIGURE 5. Graph showing the trend in mortality due to COVID-19 in the United Kingdom displayed weekly (blue line) and the surgical mortality for AAS operated on during the same period of time in the study participating centers (red line). The lines crossover on the week of the March 16, 2020, corresponding with the start of the lockdown in the United Kingdom, when the number of COVID-19 cases started to increase exponentially and the AAS activity decreased initially due to the reduced presentation to emergency departments. Both curves reached a peak around middle of April to descend in a parallel way after that. Note that the scale for the COVID-19 mortality has been adapted and has to be multiplied $\times 100$. COVID-19, Coronavirus disease 2019; AAS, acute aortic syndrome. 
operating under undue pressure, facing potential risk of devastating infection, as well as working in unfamiliar and restricted theater practices including use of full PPE. Moreover, these patients with AAS were managed in intensive care unit by staff with full PPE and were not allowed to have family members support as routine. Therefore, it was a notable achievement by the clinical teams that the clinical outcomes for patients with AAS were maintained during this period. There were a small number of patients $(\mathrm{n}=9)$ who were not treated surgically showed a high mortality $(60 \%)$ as initially expected. Once again, the reasons for turning down patients with AAS for surgery were not related to COVID-19 status or a reduction in hospital equipment or staffing but most commonly due to the complexity of the cases, with most of the patients having had previous cardiac and/or aortic surgery, making an out-of-hours redo operation very challenging with the current mandatory PPE measures.

\section{CONCLUSIONS}

The service provision for AAS was maintained during the early months of the COVID-19 pandemic in the United Kingdom by a significant restructuring of NHS, with a very low rate of surgical turn down and COVID-19-related complications. Although most patients were operated on with an unknown COVID-19 status due to the devastating natural history of the condition outweighing the risk of postoperative respiratory-related complications, only a minority of patients developed COVID-19 in the postoperative period, which did not influence outcomes. The findings from this study indicate that, with appropriate precautions, emergency surgical treatment can be provided to patients with AAS with good outcomes during the COVID-19 pandemic.

\section{Conflict of Interest Statement}

The authors reported no conflicts of interest.

The Journal policy requires editors and reviewers to disclose conflicts of interest and to decline handling or reviewing manuscripts for which they may have a conflict of interest. The editors and reviewers of this article have no conflicts of interest.

The authors acknowledge Yama Haqzad (Hull), Luke Holland and Joanne Jessup (Brighton), Yusuf Abdullahi (Hammersmith), Vamsidar Dronovalli (Birmingham), Andrew Brazier (Coventry), George Gradinariu (Aberdeen), Saif Mohamed and Deepthy
Blesson (Stoke), Marius Roman (Leicester), Anne Gregg and Ronan Kelly (Belfast), Tracey Smiles (Middlesbrough), Amanda Finch (Blackpool) for their data collection in the individual centres. Martin Yates and Julie Sanders (St Bartholomew's Hospital, London) contributed in the design of the study protocol and ethics approval.

\section{References}

1. American College of Surgeons. COVID-19: guidance for triage of nonemergence surgical procedures; Available at: www.facs.org/covid-19/clinicalguidance/triage. COVID-19 and Surgery / Clinical Issues and Guidance. Accessed March 17, 2020.

2. PCR Online. Cardiac surgery and the COVID-19 outbreak: what does it mean? Available at: www.pcronline.com/News/Whats-new-on-PCRonline/2020/Cardi ac-Surgery-and-the-COVID-19-outbreak-what-does-it-mean. Accessed March 25,2020 .

3. Hasan A, Arosra RC, Adams C, Boschard D, Cook R, Gunning D, et al. Cardiac surgery in Canada during the COVID-19 pandemic: a guidance statement from the Canadian Society of Cardiac Surgeons. Can J Cardiol. 2020;36:952-5.

4. The Vascular Society for Great Britain and Ireland. COVID-19 virus and vascular surgery; Available at: www.vascularsociety.org.uk/professinals/news/113/covid 19_virus_and_vascular_surgery. Accessed March 20, 2020.

5. Evangelista A, Isselbacher EM, Bossone E, Gleason TG, Di Eusanio M, Sechtem U, et al. Insights from the International Registry of Acute Aortic Dissection: a 20-year experience of collaborative research. Circulation. 2018;137:1846-60.

6. Society for Cardiothoracic Surgery in Great Britain \& Ireland. Barts protocol for surgery in aortovascular patients during the COVID-19 pandemic; Available at: www.scts.org/Covid-19/. Accessed April 12, 2020.

7. Gov.UK. COVID-19: guidance for health professionals from Public Health England and the Department of Health and Social Care; 2020. Available at: www gov.uk/goverment/collections/wuhan-novel-coronavirus. Accessed December $11,2020$.

8. Society for Cardiothoracic Surgery in Great Britain \& Ireland. Pan-London emergency cardiac surgery standard operating protocol during COVID-19 pandemic; Available at: www.scts.org/Covid-19/. Accessed March 27, 2020.

9. Society for Cardiothoracic Surgery in Great Britain \& Ireland. St Bartholomew's hospital theatre standard operating protocol for COVID-19; Available at: www. scts.org/Covid-19/. Accessed April 8, 2020.

10. Gov.UK. Coronavirus (COVID-19): personal protective equipment (PPE); Available at: www.gov.uk/government/coronarivrus-covid-19-personal-protecti ve-equipment-ppe. Accessed December 11, 2020.

11. Hussain A, Balmforth D, Yates M, Lopez-Marco A, Rathwell C, Lambourne J, et al. The Pan London Emergency Cardiac Surgery service: coordinating a response to the COVID-19 pandemic. J Card Surg. 2020; 35:1563-9.

12. COVIDSurg Collaborative. Mortality and pulmonary complications in patients undergoing surgery with perioperative SARS-CoV-2 infection: an international cohort study. Lancet. 2020;396:27-38.

13. Richens D. Cardiothoracic surgery GIRFT programme National specialty report Available at: www.gettingitrightfirsttime.co.uk/wp-content/uploads/2018/07/Car diothoracicReportMar18-F.pdf. Accessed May 20, 2020. 74-78.

14. Bottle A, Mariscalco G, Shaw MA, Benedetto U, Saratzis A, Mariani S, et al. Unwarranted variation in the quality of care for patients with diseases of the thoracic aorta. J Am Heart Assoc. 2017;6:e004913.

Key Words: aorta, acute aortic syndromes, emergency surgery, COVID-19 pandemic 